\title{
FORCE dual-energy CT in pathological grading of clear cell renal cell carcinoma
}

\author{
CHUNLING ZHANG ${ }^{1}$, NING WANG $^{1}$, XINYOU SU ${ }^{2}, \mathrm{KUN} \mathrm{LI}^{1}$, DEXIN YU ${ }^{3}$ and AIMEI OUYANG ${ }^{1}$ \\ Departments of ${ }^{1}$ Radiology and ${ }^{2}$ Oncology, Jinan Central Hospital, Shandong University, Jinan, Shandong 250013; \\ ${ }^{3}$ Department of Radiology, Qilu Hospital of Shandong University, Jinan, Shandong 250012, P.R. China
}

Received February 25, 2019; Accepted September 6, 2019

DOI: $10.3892 / \mathrm{ol} .2019 .11022$

\begin{abstract}
The aim of the present study was to examine the value of FORCE dual-energy CT in grading the clear cell renal cell carcinoma (ccRCC). A total of 35 cases of ccRCC were included. Hematoxylin and eosin staining was performed, and the cases were divided into low- (Fuhrman I-II) and high-grade (Fuhrman III-IV) groups. FORCE dual-energy CT parameters, including virtual network computing CT value (VNCV), iodine overlay value (IOV), mixed energy CT value (MEV), iodine concentration (IC), normalized iodine concentration (NIC), NIC based on aorta (NICA), NIC based on cortex (NICC) and NIC based on medulla (NICM), were analyzed and compared. Receiver operating characteristic analysis was also performed. There were significant differences in the arterial phase IOV, MEV and IC, and the venous phase IOV and IC between the low- and high-grade groups. No significant differences were observed in VNCV and MEV between the low -and high-grade groups in the venous phase. Significant differences were observed in the NICA and NICC between these two groups, however no difference was observed in NICM. There were significant differences in the tumor CT values for the arterial phase at the 40,60, 80 and 100 kiloelectron volt $(\mathrm{keV})$ between the low- and high-grade groups, while no significant differences were observed at the 120-140 keV levels. The k-slope for the low-grade group was significantly higher than the high-grade group. In addition, the area under curve for the arterial phase IOV, arterial phase MEV, arterial phase IC, aortic NIC, cortical NIC, venous phase IOV, venous phase IC and curve slope $\mathrm{K}$ of mono-energy CT value suggested high value in diagnosis of low- and high-grade ccRCC cases.
\end{abstract}

Correspondence to: Dr Aimei Ouyang, Department of Radiology, Jinan Central Hospital, Shandong University, 105 Jie Fang Road, Jinan, Shandong 250013, P.R. China

E-mail: 13370582510@163.com

Key words: clear cell renal cell carcinoma, FORCE dual-energy CT, Fuhrman grading

\section{Introduction}

Renal cell carcinoma originates from the renal tubule or collecting duct epithelial cells, and is one of the most common malignant tumors in the urinary system, accounting for $85 \%$ of renal malignancies (1). Clear cell renal cell carcinoma (ccRCC) is the most common renal cell carcinoma type accounting for $\sim 70 \%$ of cases (2). At present, the most widely used tumor grading system for renal carcinomas is the Fuhrman grading system, which was first proposed by Fuhrman et al in 1982 (3). According to the tumor cell nucleus size, the nucleus shape and whether the nucleoli are obvious, renal carcinoma cases could be divided into 4 grades. The Fuhrman grading system has important value in evaluating the prognosis of renal cell carcinomas $(4,5)$, representing the optimal effective indicator for predicting renal tumor invasion (6). It has been shown that there are significant differences in the survival rates between groups with high and low Fuhrman grading levels $(7,8)$. When tumor cells are divided into low (Fuhrman I-II) and high (Fuhrman III-IV) grades, satisfactory consistency may be expected. A previous study has reported that renal tumors with high Fuhrman grades are more invasive, therefore, a more rigorous follow-up plan should be implemented after kidney preservation (9).

In recent years, with the rapid development of energy spectrum CT imaging technology, CT has changed from single-parameter imaging to multi-parameter imaging, and from reflecting the gross morphology to microscopic features of lesions, with tissue specificity $(10,11)$. FORCE CT pure-spectrum photon technology enables precise separation of multi-substance components, which can automatically generate iodine- and single-energy-images, and the following parameters can be obtained: Virtual network computing (VNC) CT values, single-energy CT values, mixed energy $\mathrm{CT}$ values, iodine concentration and normalized iodine concentration (NIC) of the regions of interest (12). Previous studies have shown that the most important indicator for the differential diagnosis of ccRCCs is the degree of lesion enhancement $(13,14)$. Therefore, the lesion enhancement may be more accurately evaluated with the dual-energy parameters, in order to identify the pathological grade. It has also been applied in the diagnosis of renal carcinomas (15-17). Quantitative and semi-quantitative FORCE dual-energy CT parameters can reflect the microstructure of tumor tissue to 
a certain extent. These parameters can be used as evaluating indexes for the biological behavior of ccRCC. However, it has rarely been used in evaluating carcinomas according to the Fuhrman's grading system.

In this study, to evaluate the value of dual-energy CT in the renal carcinoma classification, the FORCE dual-energy parameters and the associated Fuhrman grading results of the ccRCCs were investigated. Dual-energy CT was used to evaluate the pathological grades of ccRCC, in order to determine the degree of malignancy based on imaging findings, to guide the choice of renal carcinoma treatment options and provide reference for treatment with nephron sparing surgery and active monitoring of renal carcinomas.

\section{Materials and methods}

Study subjects. This study included a total of 35 patients with primary single-sided ccRCC, who received abdominal FORCE dual-energy CT-enhanced scanning and had the diagnosis confirmed by surgery and pathology, between December 2017 and August 2018 in Qilu Hospital. Exclusion criteria were as follows: (i) Previous abdominal surgery, or preoperative radiotherapy, chemotherapy, and targeted therapy; (ii) cardiovascular disease and kidney disease, severely affecting the degree of kidney enhancement; and (iii) imaging showing the cystic kidney cancer with less substantial composition. Among the included patients, there were 26 males and 9 females, with an mean age of 55.1 years (range, 37-71 years). In addition, 17 cases of the cases were carcinomas of the left kidney and 18 cases were located in the right kidney, with maximum and minimum diameters of 10.1 and $1.3 \mathrm{~cm}$, respectively. Prior written and informed consent were obtained from every patient, and the study was approved by the Ethics Review Board of the Qilu Hospital.

CT scanning. CT scanning was performed with the Somatom FORCE CT machine (Siemens). Standard protocol was followed as all patients were asked to hold their breath during the scanning process, and were subjected to fasting for $4 \mathrm{~h}$ prior to the scan, as they received $500 \mathrm{ml} 5 \%$ diatrizoate before the enhanced scanning. All patients received 60-80 ml non-ionic contrast agent iohexol (300 milligrams of iodine per milliliter) by injection into the forearm with a high-pressure syringe, with the injection flow rate of $3-5 \mathrm{ml} / \mathrm{sec}$. For the arterial phase (medullary phase) and the venous phase (renal parenchyma phase), scanning delay time was set at 30 and $80 \mathrm{sec}$ after the injection of contrast agent, using the dual-energy mode, with the tube voltage of 100 kilovolt peak $(\mathrm{kVp})$ and tin filter $(\mathrm{Sn}) 150 \mathrm{kVp}$, respectively. The automatic exposure system was activated, with the tube currents of 130-180 milliampere-seconds (mAs) and 80-90 mAs, for $100 \mathrm{kVp}$ and $\mathrm{Sn} 150 \mathrm{kVp}$, respectively.

Image analysis. Images of $100 \mathrm{kVp}$ and $\mathrm{Sn} 150 \mathrm{kVp}$ were obtained from the scan, with the reconstructing layer thickness of $1.0 \mathrm{~mm}$ and layer spacing of $1.0 \mathrm{~mm}$. The image data were analyzed with the dual-energy post-processing workstation (syngo.via; version VB10; Siemens Healthineers). The iodine maps and virtual network computing images for the arterial and venous phases were obtained using the Liver VNC (18) method. Based on the iodine maps, the VNC CT value (VNCV), iodine overlay value (IOV), mixed energy CT value
(MEV), iodine concentration (IC), and the tumor IC difference of artery phase and venous phase (DIC) were measured and recorded. The NIC was measured by the normalization method, including the NIC based on aorta (NICA), NIC based on cortex (NICC) and NIC based on medulla (NICM). The single-energy $\mathrm{CT}$ values and the tumor energy spectrum for the arterial phase of the 40, 60, 80, 100, 120 and 140 kiloelectron volt $(\mathrm{keV})$ were obtained with the single-energy software. The slope of the energy spectrum curve was calculated using the following formula: $\mathrm{k}=$ [hounsfield unit (HU)40 keV-HU100 keV]/100 keV-40 keV. The CT images were independently analyzed, measured and recorded by two senior imaging diagnosticians in Qilu Hospital with the PACS system (Agfa Graphics Ltd.) and the FORCE CT workstation, in a blinded manner in terms of the surgical and pathological results. The area of interest was selected to be as large as possible where the necrotic areas and blood vessels were avoided. The data obtained were comprehensively compared and the consistency of the researchers was performed using the Kappa-test. Two different technologists read the measurements, and if their opinions were different, a third technologist should be involved to settle the discrepancy. The measurement was conducted again based on the discussion and negotiation.

Hematoxylin and eosin (HE) staining. The surgical ccRCC tissures were fixed in $10 \%$ formalin at room temperature for 6-48 h, and the gross pathological condition was observed. After paraffin embedding at $60^{\circ} \mathrm{C}$ for $4 \mathrm{~h}$, the specimens were cut into $4-\mu \mathrm{m}$ continuous sections, which were subjected to HE staining (Fuzhou Maixin Biotech Co., Ltd.).

Pathological image analysis. The pathological images were analyzed under light microscope (magnification, x100; Olympus Corporation) by two experienced pathologists from Qilu Hospital in a blinded manner in terms of CT findings. The specimens diagnosed as ccRCC were graded using the following Fuhrman grading system: Grade I, small and round nucleus, diameter $10 \mu \mathrm{m}$, and size similar to the red blood cells, with uniform size and no obvious nucleoli; grade II, slightly irregular nucleus (nucleoli obvious), with the same size and diameter of $15 \mu \mathrm{m}$; grade III, irregular nucleus (nucleoli large and obvious), diameter $20 \mu \mathrm{m}$; and grade IV, singular nucleus (or lobulated), diameter $\sim 25 \mu \mathrm{m}$ and large nucleoli. Using HE staining, renal carcinomas with the Fuhrman grades of I-II and grades III-IV were classified as the low-grade group and the high-grade group, respectively.

Statistical analysis. Data were expressed as mean \pm standard error. Statistical analysis was performed using the SAS 9.2 software (SAS Institute, Inc.). After the Shapiro-Wilk normality test, the unpaired Student's t-test was performed for group comparison. Receiver operator characteristic curve (ROC) analysis was performed, and the area under curve (AUC), sensitivity and specificity of each index were analyzed. $\mathrm{P}<0.05$ was considered to indicate a statistically significant difference.

\section{Results}

Dual-energy CT performance. Dual-energy CT performance was first analyzed, and among these 35 patients with 
ccRCC, the largest cross-sectional length of the tumor ranged between 1.3 and $10.1 \mathrm{~cm}$. There were 22 cystic cases and 20 pseudo-enveloped cases. The pseudo-enveloped cases were persistently mild or moderately enhanced, and the necrotic cystic area was not enhanced. In 17 cases, the lesions were located in the left kidney, and in the remaining 18 cases, the lesions were located in the right kidney. Out of the 35 cases, 24 cases had a maximum cross-sectional long diameter $>4 \mathrm{~cm}$, and in these 24 patients, there were 6 cases with a maximum cross-sectional long diameter $>7 \mathrm{~cm}$. On the other hand, there were 29 cases with a maximum cross-sectional diameter $<7 \mathrm{~cm}$, and in those patients, there were 11 cases with the maximum cross-sectional diameter $<4 \mathrm{~cm}$. Furthermore, in these 35 patients, there were 31 with uneven enhancement, including necrotic cystic changes, hemorrhagic non-enhanced areas and obvious blood supply, and 4 patients with even enhancement, which was defined as the difference between the highest and lowest CT values in tumor $<20 \mathrm{HU}$.

In addition, the majority of the ccRCC tumor was enhanced for the arterial phase, with a red iodine map (partially bright orange), similar to the renal cortex (Fig. 1A-C), which was reduced during the venous phase (Fig. 1D). The single-energy image showed that, along with the increased single-energy $\mathrm{keV}$, the single-energy $\mathrm{CT}$ value of the tissue was gradually decreased, and the difference in CT values between tissues became smaller; while with decreased single-energy $\mathrm{keV}$, the CT value of the tissue was gradually increased, and the difference in $\mathrm{CT}$ values between tissues became larger (Fig. 2). These results suggest that, the tissue resolution would be elevated along with the increasing $\mathrm{keV}$ level.

Pathological results. The pathological findings were subsequently investigated. The results showed that the tumor surface was light yellow (or grayish white), and the sectional surface could be multicolored. The non-tumor surface had a flesh-pink coloring. In some cases, the tumors compressed the surrounding tissue to form a pseudo-envelope. Changes like focal necrosis, hemorrhage and calcification were commonly observed.

Among the 35 cases of ccRCCs, there were 2 cases of Fuhrman grade I, 25 cases of grade II, 7 cases of grade III and 1 case of grade IV. Therefore, there were 27 cases in the low-grade group and 8 cases in the high-grade group. Focal necrosis, hemorrhage and calcification were found in 7,1 and 6 cases respectively. These results indicate that ccRCCs are rich in blood supply, with great heterogeneity.

Dual-energy CT iodine map index for ccRCCs. The Kappa test showed that the two radiologists' measurement results were consistent. The results of the t-test comparing the dual-energy CT iodine map index between low- and high-grade ccRCCs are presented in Table I, and the mean values of the dual-energy CT iodine map index for the 35 cases of ccRCCs are shown in Table II. The dual-energy CT parameters for the venous period in the low-grade group, including the IOV, MEV and IC, were greater than the high-grade group. Among these indicators, significant differences were observed in the IOV, MEV and IC for the arterial phase, and the IOV and IC for the venous phase between the low- and high-grade groups $(\mathrm{P}<0.01)$. In addition, there was no significant difference in the VNCV for both phases and the MEV for venous phase between the two groups $(\mathrm{P}>0.05)$. These results suggest that arterial IOV, MEV and IC, as well as venous IOV and IC, have certain values in the identification of high and low grades of ccRCC.

FORCE CT-normalized index for $c c R C C$. The results of the t-test comparing the FORCE CT-normalized index for low- and high-grade ccRCCs are presented in Table II. The results showed that the NICA and NICC values for the arterial phase in the low-grade group were significantly higher than the high-grade group $(\mathrm{P}<0.01)$. However, no significant difference was observed in the NICM between the low- and high-grade groups $(\mathrm{P}>0.05)$. NICA and NICC values for the arterial phase were used to identify the pathological grades of renal tumors, while the value of NICM was not significant.

FORCE dual-energy CT single-energy index for ccRCCs. The results of the t-test comparing the FORCE dual-energy CT single-energy index for low- and high-grade ccRCCs, and the single-energy CT values for the arterial phase (VTA) at $40-140 \mathrm{keV}$ for these 35 cases are presented in Table III. These results revealed that the CT value for the arterial phase peaked at $40 \mathrm{keV}$. In addition, with increase $\mathrm{keV}$, the VTA decreased, and significant differences were observed in the VTA at the 40, 60, 80 and $100 \mathrm{keV}$ levels between the low- and high-grade groups $(\mathrm{P}<0.05)$, while no significant differences were observed in the VTA at 120-140 keV levels between these two groups $(\mathrm{P}>0.05)$. Furthermore, the mean k-slopes of the tumor energy spectrum curves for the low- and high-grade groups were $4.76 \pm 0.36$ and $2.65 \pm 0.60$, respectively, and the k-slope for the low-grade group was significantly higher than the low-grade group $(\mathrm{P}<0.05)$. These results suggest that the arterial phase CT value and the energy spectrum curve slope $(\mathrm{K})$ at low $\mathrm{keV}$ level (40-100 keV) can be used as an evaluation index for renal tumor grading.

ROC curve analysis of dual-energy CT parameters for $c c R C C s$. Results from the ROC curve analysis of dual-energy CT parameters are presented in Fig. 2 and Table IV. The results indicated that the AUC values for the arterial phase IOV, arterial phase MEV, arterial phase IC, aortic NIC, cortical NIC, venous phase IOV, venous phase IC and curve slope $\mathrm{K}$ were $0.81,0.78,0.80,0.76,0.80,0.80,0.81$ and 0.79 , respectively. This suggests that the aforementioned indicators may be of high value for the identification of high- and low-grade ccRCCs. Among these indicators, the diagnostic cut-off value for arterial phase IOV was 57.30, with sensitivity and specificity of 0.86 and 0.75 , respectively. The diagnostic cut-off value for arterial phase IC was 2.65 , with sensitivity and specificity of 0.76 and 0.75 , respectively. The diagnostic cut-off value for cortical NIC was 0.66 , with sensitivity and specificity of 0.76 and 0.88 , respectively. The sensitivity and specificity of IOV were 0.86 and 0.75 , respectively, and the sensitivity and specificity of IC were 0.76 and 0.75 , respectively. The diagnostic cut-off value for curve slope $\mathrm{K}$ was 3.04, with sensitivity and specificity of 0.76 and 0.75 , respectively. These results suggest that dual-energy $\mathrm{CT}$ parameters, including IOV, IC, NIC and K, have certain value in the identification of high- and low-grades of ccRCCs. 

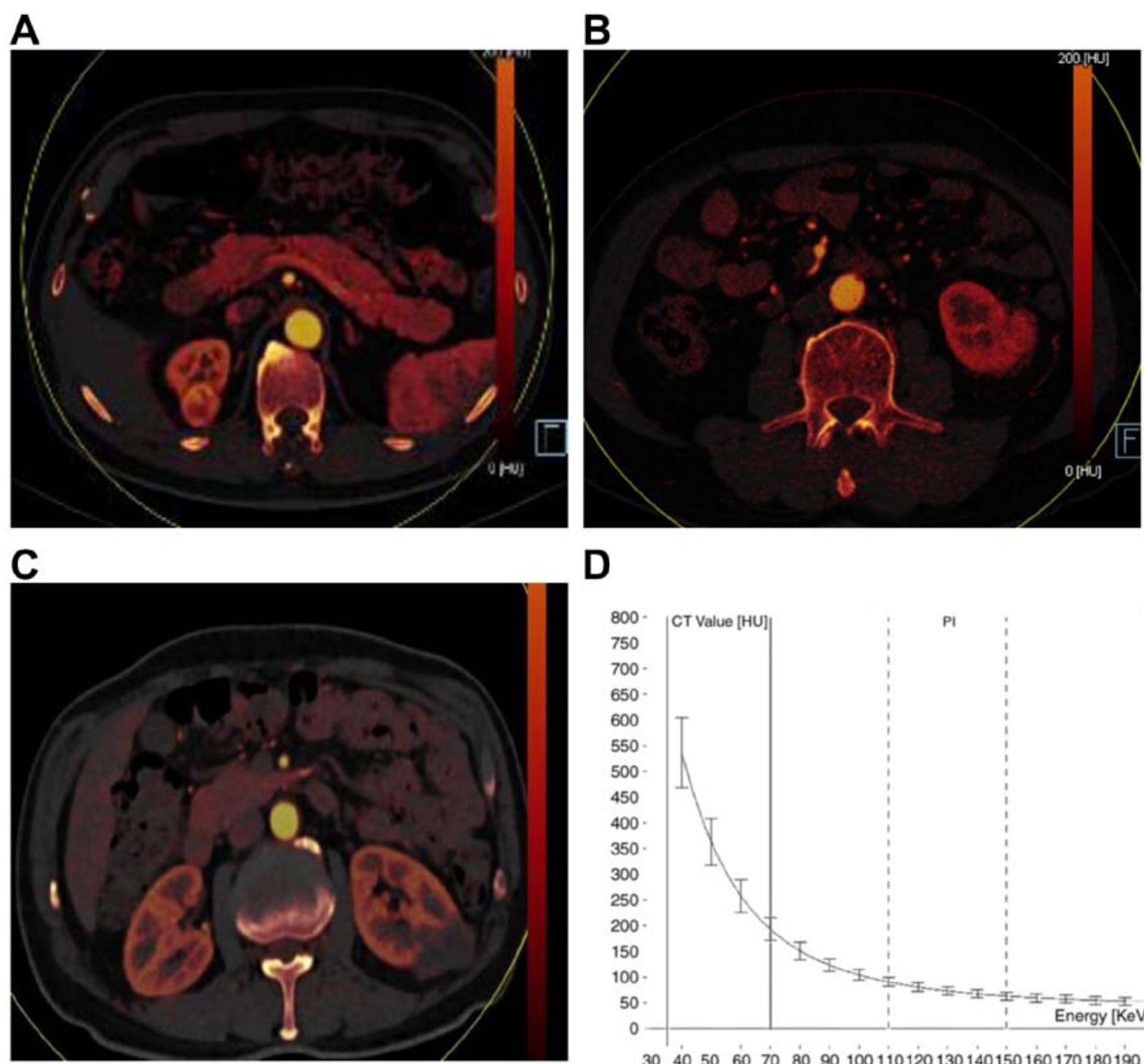

D

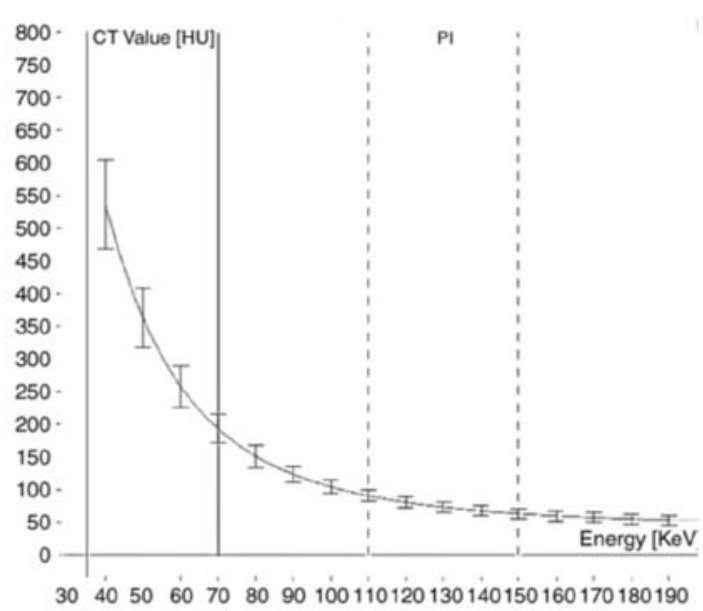

Figure 1. Iodine map and energy spectrum curves for FORCE dual-energy CT cases. (A) A 57-year-old male case of Fuhrman grade I ccRCC. The tumor enhancement degree was higher than that of the renal cortex, showing as orange on the iodine map. (B) A 69-year-old male case of Fuhrman grade II ccRCC. The enhancement degree of tumor was close to that of the renal cortex (red on iodine map). (C) A 57-year-old male case of Fuhrman grade IV ccRCC. The tumor enhancement degree was mild and lighter than that of renal cortex (gray on iodine map). (D) The related energy spectrum from the subject presented in part B. ccRCC, clear cell renal cell carcinoma.

\section{Discussion}

With the development of imaging technology, energy spectrum CT imaging has gradually become a research hotspot, and this has transformed CT from single-parameter imaging to multi-parameter imaging (19). Through unique energy spectrum scanning and image post-processing technology, a wider range of lesions with multiple parameters can be identified and quantitative analysis can be performed (20). Therefore, CT reflects the gross morphology of the lesions and the macroscopic features, which may serve a prospective role in objectively assessing the tissue characteristics and functional status of the lesions (21-23).

FORCE dual-energy CT represents an extremely advanced spiral CT, with high density and time resolution $(24,25)$. In addition to two- and three-dimensional recombination like ordinary spiral CT, its unique pure-spectrum photon energy purification technology enables dual-energy imaging, which allows for more accurate energy-and-material separation (10). Using spectral purification technology, the radiation dose can be effectively reduced while meeting the diagnostic requirements. During only one scanning event, the parameters, such as the VNCV, IOV, MEV, IC, NIC and CT values at different single-energy levels, can be obtained, and this study speculated that these parameters are not interfered by normal affecting factors. Therefore, the technology may allow more precise measurements of lesion iodine uptake, thereby contributing quantitative determination of the enhancement degree of tissue and organs, as well as the blood supply, which effectively reflects the microstructure and hemodynamic changes of tumor tissues (26). This information may assist in obtaining a more accurate and comprehensive qualitative and quantitative disease diagnosis.

Dual-energy CT iodine quantification has been applied in the differential diagnosis of benign and malignant renal tumors for tumor classification, and the majority of the machines are less advanced dual-energy CT (10-12). FORCE dual-energy CT allows pathological grading of renal tumors by quantitative determination of substance and energy separately. In the present study, the results showed that the AUC 
Table I. Dual-energy CT iodine map indicators for low- and high-grade clear cell renal cell carcinomas.

\begin{tabular}{|c|c|c|c|c|}
\hline Dual-energy CT iodine map indicators & Low-grade, $n=27$ & High-grade, $n=8$ & t-test & P-value \\
\hline VNCV & $35.82 \pm 1.44$ & $37.76 \pm 1.84$ & -0.68 & 0.501 \\
\hline Arterial phase IOV & $93.96 \pm 7.45$ & $48.86 \pm 10.57$ & 3.03 & 0.005 \\
\hline Arterial phase MEV & $129.60 \pm 7.46$ & $87.88 \pm 11.28$ & 2.77 & 0.009 \\
\hline Arterial phase IC & $3.98 \pm 0.28$ & $2.13 \pm 0.44$ & 3.25 & 0.003 \\
\hline Venous phase IOV & $75.57 \pm 5.33$ & $43.47 \pm 6.95$ & 3.04 & 0.005 \\
\hline Venous phase IC & $3.35 \pm 0.26$ & $1.93 \pm 0.31$ & 3.13 & 0.004 \\
\hline $\mathrm{DIC}$ & $0.39 \pm 0.16$ & $0.20 \pm 0.28$ & 0.62 & 0.529 \\
\hline
\end{tabular}

VNCV, virtual network computing CT value; IOV, iodine overlay value; MEV, mixed energy CT value; IC, iodine concentration; DIC, tumor IC difference of artery phase and venous phase.

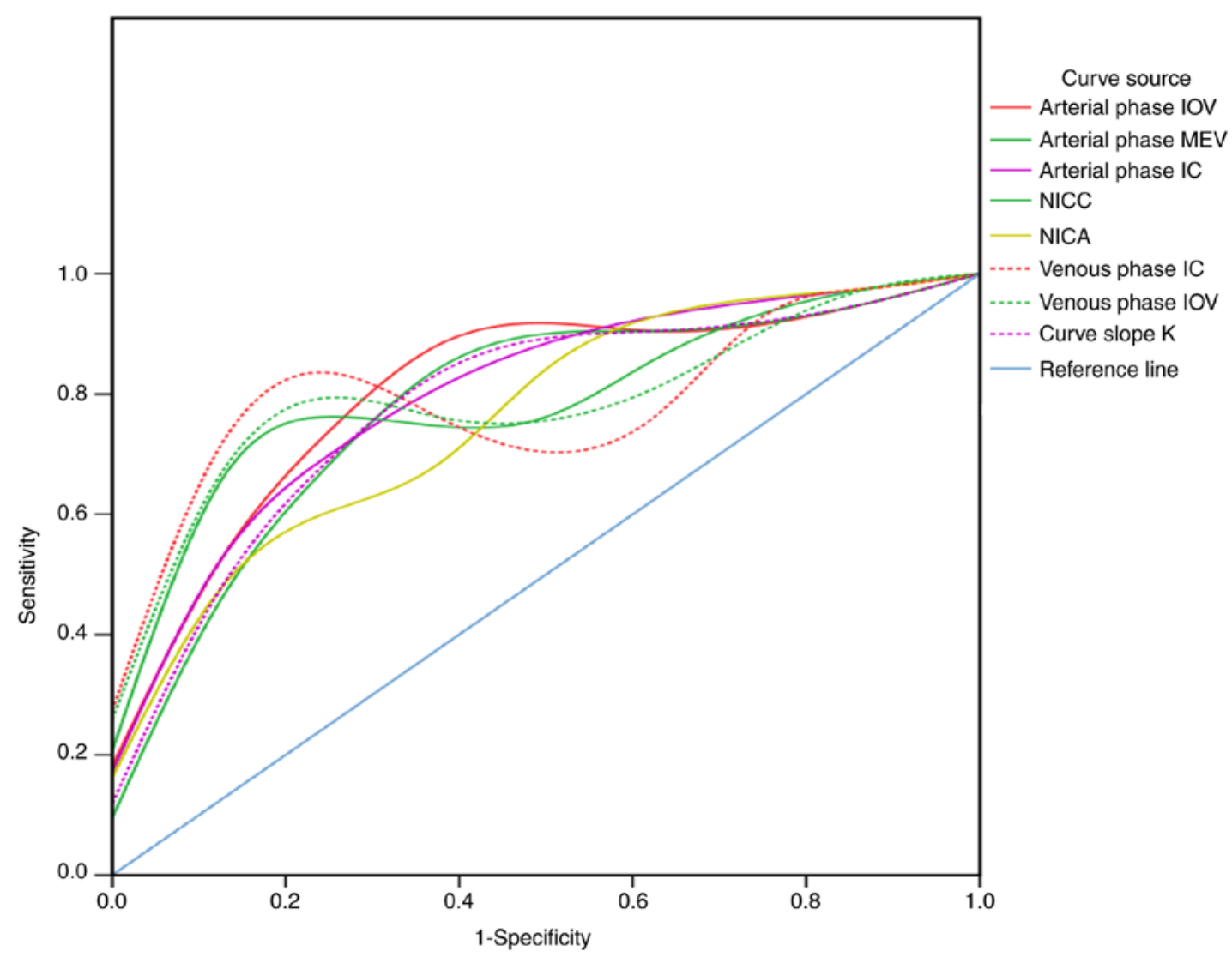

Figure 2. Receiver operator characteristic curve analysis for FORCE double energy parameter in ccRCC grading. ccRCC, clear cell renal cell carcinoma; NIC, normalized iodine concentration; NICA, NIC based on aorta; NICC, NIC based on cortex; IOV, iodine overlay value; MEV, mixed energy CT value; IC, iodine concentration.

for arterial phase IOV, arterial phase MEV, arterial phase IC, aortic NIC, cortical NIC, venous phase IOV, venous phase IC and curve slope $\mathrm{K}$ of high- and low-grade renal cell carcinomas were $0.810,0.780,0.804,0.762,0.804,0.798,0.807$ and 0.786 , respectively, suggesting high value for the pathological grading of renal tumors. High grade ccRCC cases had low levels of IOV and IC values, which was in accordance with previous literature (27-29). Among these indicators, the AUC of the arterial phase IOV was the largest, with the diagnostic cut-off value of 57.30, and sensitivity and specificity of 0.857 and 0.750 , respectively. The reason for this may be that the degree of tumor enhancement was associated with the vascularization status (30-32). The lower grade was associated with mature microvessels and therefore, an effective blood supply could be transported to the tumor, resulting in larger IC and IOC values. In contrast, the higher grade was associated with less mature microvessels and lower lumenization level, with an insufficient blood supply leading to lower levels of IC and IOC values. In addition, the high-grade tumors had immature vessels with high permeability, which may lead to increased blood viscosity and interstitial osmotic pressure, as one of the reasons for the decreased blood flow velocity (27). In order 
Table II. FORCE dual-energy CT normalized parameters for low- and high-grade clear cell renal cell carcinomas.

\begin{tabular}{lccr}
\hline CT normalized parameters & Low-grade, $n=27$ & High-grade, $n=8$ & t-test \\
\hline NICA & $0.33 \pm 0.023$ & $0.19 \pm 0.04$ & 2.92 \\
NICC & $0.80 \pm 0.05$ & $0.48 \pm 0.09$ & 3.03 \\
NICM & $3.60 \pm 0.31$ & $3.23 \pm 0.55$ & 0.006 \\
\hline
\end{tabular}

NIC, normalized iodine concentration; NICA, NIC based on aorta; NICC, NIC based on cortex; NICM, NIC based on medulla.

Table III. FORCE CT single-energy indicator (VTA, k) for low- and high-grade clear cell renal cell carcinomas.

\begin{tabular}{lcccr}
\hline Single-energy, $\mathrm{keV}$ & Low-grade, $\mathrm{n}=27$ & High-grade, $\mathrm{n}=8$ & $\mathrm{t}$ & P-value \\
\hline 40 & $366.70 \pm 24.66$ & $221.50 \pm 41.46$ & 2.85 & 0.007 \\
60 & $180.90 \pm 10.91$ & $119.00 \pm 18.40$ & 2.76 & 0.009 \\
80 & $110.80 \pm 5.81$ & $79.69 \pm 9.59$ & 2.62 & 0.001 \\
100 & $80.54 \pm 3.52$ & $62.60 \pm 5.77$ & 2.49 & 0.018 \\
120 & $63.53 \pm 2.51$ & $53.96 \pm 3.92$ & 1.88 & 0.069 \\
140 & $54.73 \pm 1.97$ & $48.94 \pm 2.73$ & 1.48 & 0.149 \\
160 & $50.03 \pm 1.72$ & $46.31 \pm 2.39$ & 1.08 & 0.290 \\
$\mathrm{k}$ & $4.76 \pm 0.36$ & $2.65 \pm 0.60$ & 2.88 & 0.007 \\
\hline
\end{tabular}

Table IV. Receiver operator characteristic curve analysis of dual-energy CT parameters.

\begin{tabular}{lcccc}
\hline CT parameters & AUC & Cut-off value & Sensitivity & Specificity \\
\hline Arterial phase IOV & 0.81 & 57.30 & 0.86 & 0.75 \\
Arterial phase MEV & 0.78 & 97.35 & 0.76 & 0.75 \\
Arterial phase IC & 0.80 & 2.65 & 0.76 & 0.75 \\
NICA & 0.76 & 0.21 & 0.91 & 0.50 \\
NICC & 0.80 & 0.66 & 0.76 & 0.88 \\
Venous phase IOV & 0.80 & 61.60 & 0.76 & 0.88 \\
Venous phase IC & 0.81 & 2.70 & 0.76 & 0.88 \\
Curve slope K & 0.79 & 3.04 & 0.76 & 0.75 \\
\hline
\end{tabular}

AUC, area under the curve; IOV, iodine overlay value; MEV, mixed energy CT value; IC, iodine concentration; NIC, normalized iodine concentration; NICA, NIC based on aorta; NICC, NIC based on cortex.

to correct the system iodine load error, the relative iodine concentration ratios were also used for the dual-energy CT parameters, including the NIC for the tumor compared with the aorta, cortex and medulla. These results showed that there was statistical significance for the normalized NIC values for the tumor based on aorta and cortex, with the AUC values of 0.762 and 0.804 , respectively, suggesting their diagnostic value for the high- and low-grade renal cell carcinomas.

The findings of the present study indicated that for the 40-100 keV single-energy levels, significant differences were observed in the tumor CT value for the arterial phase between the low- and high-grade groups, while there was no significant difference in the CT value at the 120-160 single-energy levels. The reason may be that higher keV resulted in decreased CT values of the tissues, and less significant differences in $\mathrm{CT}$ values between tissues, and between low- and high-grade groups. Through the analysis of the tumor energy spectrum curve, the results showed that the slope of the energy spectrum curve peaked at the $40-100 \mathrm{keV}$ levels. Therefore, the following curve slope $\mathrm{k}$ used herein was calculated according to the following formulation: $\mathrm{k}=\left(\mathrm{HU}_{40 \mathrm{keV}}-\mathrm{HU}_{100 \mathrm{keV}}\right) / 100 \mathrm{keV}-$ $40 \mathrm{keV}=\left(\mathrm{HU}_{40 \mathrm{keV}}-\mathrm{HU}_{100 \mathrm{keV}}\right) / 60 \mathrm{keV}$. The results showed that the AUC value for the k-value in diagnosing advanced ccRCC cases was 0.78 , with a diagnostic cut-off value of 3.04 , and sensitivity and specificity of 0.76 and 0.75 , respectively, which could be applied as a useful diagnostic indicator. These results suggest that the curve slope $\mathrm{k}$ has certain value in the pathological grading of renal tumors.

FORCE dual-energy CT can provide higher accuracy when reflecting the renal cancer microstructure, with better 
diagnostic value compared with conventional enhanced CT. The dual-energy parameters have certain tissue specificity, which can accurately reflect the tissue composition (10). However, the iodine map could accurately extract the iodine representing the enhancement degree, so as to objectively and accurately reflect the blood supply and microvessels for the tumors (19). There are certain limitations to the current study, including a limited sample size of dual-energy CT-examined cases and a lack of analysis of other types of renal tumors. Further in-depth studies are required to address these issues.

Quantitative and semi-quantitative FORCE dual-energy CT parameters may reflect the microstructure of tumor tissues to a certain extent. The findings of the present study suggest that these parameters could be used as an index to evaluate the biological behavior of ccRCCs, contributing to clinical treatment choice and prognostic assessment.

\section{Acknowledgements}

Not applicable.

\section{Funding}

The present study was supported by the Fundamental Research Funds of Shandong University (grant no. 2014QLKY01).

\section{Availability of data and materials}

The datasets used and/or analyzed during the present study are available from the corresponding author on reasonable request.

\section{Authors' contributions}

AO designed the study and collected the funds. CZ and NW collected the data. XS and KL performed the statistical analysis and drafting. AO and DY interpreted the data. KL and DY searched the literature. $\mathrm{CZ}$ and KL prepared the manuscript. All authors read and approved the final manuscript.

\section{Ethics approval and consent to participate}

Prior written and informed consent were obtained from every patient. The present study was approved by the Ethics Review board of Qilu Hospital, Shandong University.

\section{Patient consent for publication}

Not applicable.

\section{Competing interests}

The authors declare that they have no competing interests.

\section{References}

1. Kim JK, Kim TK, Ahn HJ, Kim CS, Kim KR and Cho KS Differentiation of subtypes of renal cell carcinoma on helical CT scans. AJR Am J Roentgenol 178: 1499-1506, 2002.

2. Hu Y, Lu J, Li Y, et al: Expression level of lncRNA CCAT2 is related with the clinical stage of RCC. Chinese J Clin, 2015 (In Chinese).
3. Fuhrman SA, Lasky LC and Limas C: Prognostic significance of morphologic parameters in renal cell carcinoma. Am J Surg Pathol 6: 655-663, 1982

4. Ljungberg B, Cowan NC, Hanbury DC, Hora M, Kuczyk MA, Merseburger AS, Patard JJ, Mulders PF and Sinescu IC; European Association of Urology Guideline Group: EAU guidelines on renal cell carcinoma: The 2010 Update. Eur Urol 58: 398-406, 2010.

5. Lohse CM and Cheville JC: A review of prognostic pathologic features and algorithms for patients treated surgically for renal cell carcinoma. Clin Lab Med 25: 433-464, 2005.

6. Teng J, Gao Y, Chen M, Wang K, Cui X, Liu Y and Xu D: Prognostic value of clinical and pathological factors for surgically treated localized clear cell renal cell carcinoma. Chin Med J (Engl) 127: 1640-1644, 2014.

7. Lang H, Lindner V, de Fromont M, Molinié V, Letourneux H, Meyer N, Martin M and Jacqmin D: Multicenter determination of optimal interobserver agreement using the Fuhrman grading system for renal cell carcinoma: Assessment of 241 patients with >15-year follow-up. Cancer 103: 625-629, 2005.

8. Wang R, Wolf JS Jr, Wood DP Jr, Higgins EJ and Hafez KS: Accuracy of percutaneous core biopsy in management of small renal masses. Urology 73: 581-590, 2009.

9. Lin RF: Predictors of recurrence after nephron-sparing surgery for renal cell carcinoma. PhD dissertation, Fujian Medical University, 2017.

10. Punjabi GV: Multi-energy spectral CT: Adding value in emergency body imaging. Emerg Radiol 25: 197-204, 2018.

11. Li Z, Leng S, Yu L, Manduca A and McCollough CH: An effective noise reduction method for multi-energy $\mathrm{CT}$ images that exploits spatio-spectral features. Med Phys 44: 1610-1623, 2017.

12. Zhou W, Schornak R, Michalak G, Weaver J, Abdurakhimova D, Ferrero A, Fetterly KA, McCollough $\mathrm{CH}$ and Leng S: Determination of optimal image type and lowest detectable concentration for iodine detection on a photon counting detector-based multi-energy CT System. Proc SPIE Int Soc Opt Eng 10573: 105734U, 2018.

13. Choi SY, Sung DJ, Yang KS, Kim KA, Yeom SK, Sim KC, Han NY, Park BJ, Kim MJ, Cho SB and Lee JH: Small $(<4 \mathrm{~cm})$ clear cell renal cell carcinoma: Correlation between CT findings and histologic grade. Abdom Radiol (NY) 41: 1160-1169, 2016.

14. Coy H, Young JR, Douek ML, Pantuck A, Brown MS, Sayre J and Raman SS: Association of qualitative and quantitative imaging features on multiphasic multidetector CT with tumor grade in clear cell renal cell carcinoma. Abdom Radiol (NY) 44: 180-189, 2018.

15. Brown CL, Hartman RP, Dzyubak OP, Takahashi N, Kawashima A, McCollough CH, Bruesewitz MR, Primak AM and Fletcher JG: Dual-energy CT iodine overlay technique for characterization of renal masses as cyst or solid: A phantom feasibility study. Eur Radiol 19: 1289-1295, 2009.

16. Chandarana H, Megibow AJ, Cohen BA, Srinivasan R, Kim D, Leidecker C and Macari M: Iodine quantification with dual-energy CT: Phantom study and preliminary experience with renal masses. AJR Am J Roentgenol 196: W693-W700, 2011.

17. Mileto A, Nelson RC, Samei E, Jaffe TA, Paulson EK, Barina A, Choudhury KR, Wilson JM and Marin D: Impact of dual-energy multi-detector row $\mathrm{CT}$ with virtual monochromatic imaging on renal cyst pseudoenhancement: In vitro and in vivo study. Radiology 272: 767, 2014.

18. Sauter AP, Muenzel D, Dangelmaier J, Braren R, Pfeiffer F, Rummeny EJ, Noël PB and Fingerle AA: Dual-layer spectral computed tomography: Virtual non-contrast in comparison to true non-contrast images. Eur J Radiol 104: 108-114, 2018.

19. McCollough CH, Leng S, Yu L and Fletcher JG: Dual- and multi-energy CT: Principles, technical approaches, and clinical applications. Radiology 276: 637-653, 2015.

20. Grajo JR, Patino M, Prochowski A and Sahani DV: Dual energy CT in practice: Basic principles and applications. Appl Radiol 45: 6-12, 2016.

21. Wang G, Zhang C, Li M, Deng K and Li W: Preliminary application of high-definition computed tomographic Gemstone Spectral Imaging in lung cancer. J Comput Assist Tomogr 38: 77-81, 2014.

22. Wei J, Zhao J, Zhang X, Wang D, Zhang W, Wang Z and Zhou J: Analysis of dual-energy spectral CT and pathological grading of clear cell renal cell carcinoma (ccRCC). PLoS One 13: e0195699, 2018.

23. Hou WS, Wu HW, Yin Y, Cheng JJ, Zhang Q and Xu JR: Differentiation of lung cancers from inflammatory masses with dual-energy spectral CT Imaging. Acad Radiol 22: 337-344, 2015. 
24. Weis M, Henzler T, Nance JW Jr, Haubenreisser H, Meyer M, Sudarski S, Schoenberg SO, Neff KW and Hagelstein C: Radiation dose comparison between $70 \mathrm{kVp}$ and $100 \mathrm{kVp}$ with spectral beam shaping for non-contrast-enhanced pediatric chest computed tomography: A prospective randomized controlled study. Invest Radiol 52: 155-162, 2017.

25. Agliata G, Schicchi N, Agostini A, Fogante M, Mari A, Maggi S and Giovagnoni A: Radiation exposure related to cardiovascular CT examination: Comparison between conventional 64-MDCT and third-generation dual-source MDCT. Radiol Med 124: 753-761, 2019.

26. Kaltenbach B, Wichmann JL, Pfeifer S, Albrecht MH, Booz C, Lenga L, Hammerstingl R, D'Angelo T, Vogl TJ and Martin SS Iodine quantification to distinguish hepatic neuroendocrine tumor metastasis from hepatocellular carcinoma at dual-source dual-energy liver. CT Eur J Radiol 105: 20-24, 2018.

27. Li X, Jiang R and Wang B: Differentiation of renal clear cell carcinoma: Evaluation with dual-source CT iodine quantification. J Clin Radiol 35: 1542-1545, 2016.

28. Wei J, Zhao J, Zhang X, Wang D, Zhang W, Wang Z and Zhou J: Analysis of dual energy spectral CT and pathological grading of clear cell renal cell carcinoma (ccRCC). PLoS One 13: e0195699, 2018.
29. Zhao J,Zhang P, Chen X, Cao W and Ye Z: Lesion size and iodine quantification to distinguish low-grade from high-grade clear cell renal cell carcinoma using dual-energy spectral computed tomography. J Comput Assist Tomogr 40: 673-677, 2016.

30. Ouyang AM, Wei ZL, Su XY, Li K, Zhao D, Yu DX and Ma XX: Relative computed tomography (CT) enhancement value for the assessment of microvascular architecture in renal cell carcinoma. Med Sci Monit 23: 3706-3714, 2017

31. Jia ZZ, Gu HM, Zhou XJ, Shi JL, Li MD, Zhou GF and Wu XH: The assessment of immature microvascular density in gliomas with dynamic contrast-enhanced magnetic resonance imaging. Eur J Radiol 84: 1805-1809, 2015.

32. Xiong Z, Liu JK, Hu CP, Zhou H, Zhou ML and Chen W: Role of immature microvessels in assessing the relationship between CT perfusion characteristics and differentiation grade in lung cancer. Arch Med Res 41: 611-617, 2010.

cc) (7) $($ This work is licensed under a Creative Commons

EY NG ND Attribution-NonCommercial-NoDerivatives 4.0 International (CC BY-NC-ND 4.0) License. 